\title{
Catgut Implantation at Acupoint Reduces Immune Reaction in a Rat Model of Allergic Rhinitis
}

\author{
Shasha Yang, ${ }^{1}$ Jing Wu, ${ }^{2}$ Qinxiu Zhang $\mathbb{D},{ }^{3}$ Xinrong Li, ${ }^{3}$ Daien Liu, ${ }^{1}$ Bin Zeng, \\ Hongjiao Gao, ${ }^{1}$ Xiaolin Yan, ${ }^{1}$ and Zhendong Zhong ${ }^{4}$ \\ ${ }^{1}$ Chengdu University of Traditional Chinese Medicine, Chengdu, Sichuan 610072, China \\ ${ }^{2}$ Guiyang College of Traditional Chinese Medicine, Guiyang, Guizhou 550002, China \\ ${ }^{3}$ Department of Otorhinolaryngology, Head and Neck Surgery of the Teaching Hospital of Chengdu University of \\ Traditional Chinese Medicine, Chengdu, Sichuan 610072, China \\ ${ }^{4}$ Sichuan Academy of Medical Sciences and Sichuan Provincial People's Hospital, Chengdu 610072, China
}

Correspondence should be addressed to Qinxiu Zhang; zhqinxiu@163.com

Received 12 December 2017; Accepted 7 June 2018; Published 5 July 2018

Academic Editor: Hyunsu Bae

Copyright (C) 2018 Shasha Yang et al. This is an open access article distributed under the Creative Commons Attribution License, which permits unrestricted use, distribution, and reproduction in any medium, provided the original work is properly cited.

\begin{abstract}
Allergic rhinitis (AR), an IgE-mediated response, is characterized by a Th2-type immunological pattern together with mast cells activation. Acupuncture, with the use of implanted catgut, is a traditional therapy that has been widely applied for the treatment of AR. However, the exact mechanism of the immunomodulatory effects of catgut implantation at acupoint (CIAA) remains unclear, in part due to the lack of a suitable laboratory animal model. We developed and optimized a rat model of ovalbumin- (OVA-) induced allergic inflammation, characterized by increased IL-4, sIgE, and SP and reciprocal decrease of IFN- $\gamma$. In the present study, we have further used this model to address the immunomodulatory effects of CIAA stimulation at Yingxiang (LI20) and Zusanli (ST36) acupoints and to elucidate the mechanisms involved in the regulation of SP, sIgE, IL-4, IFN- $\gamma$, TLR2, and TLR4. After AR model was established via OVA challenge, the rats were randomized as follows: control, model, sham-operated, 1-week CIAA (C1), 2-week CIAA (C2), and Budesonide nasal spray. The $\mathrm{C} 1$ and $\mathrm{C} 2$ groups were subjected to the bilateral acupoint Yingxiang (LI20) and Zusanli (ST36), respectively. Multiple analyses and quantifications were performed, which revealed that due to the persistent stimulus to acupoints by embedding catgut, the $\mathrm{C} 2$ group improved $\mathrm{AR}$ symptoms, compared to the $\mathrm{C} 1$ group. We conclude that CIAA at the Yingxiang (LI20) and Zusanli (ST36) acupoints effectively reduces allergic symptoms and inflammatory parameters in the rat model of AR. Thus, CIAA treatment is potentially an alternative therapeutic modality in AR.
\end{abstract}

\section{Introduction}

Allergic rhinitis (AR) is an IgE-mediated response, characterized by Th2 immunological pattern, mainly occurring together with mast cells activation [1], thus inducing sneezing, itching, nasal congestion, watery rhinorrhea, and even impaired quality of life (QOL) [2-5]. AR is a highly prevalent chronic disease that affects approximately $40 \%$ of the world's population [6]. In China, a cross-population study, conducted in 11 major cities, indicated that the prevalence of AR ranges from $8.7 \%$ to $24.1 \%$ [7]. Although Intranasal corticosteroids and antihistamines exhibit clinical efficacy for the treatment of AR [8], they cause inevitable side effects such as hormone resistance, drowsiness, and sedation. Thus, finding an effective treatment of AR has remained a major challenge.

Acupuncture is a traditional therapy that has been widely applied for the treatment of AR. In 2015, acupuncture treatment was incorporated into the United States guidelines for the treatment of AR [9]. Acupuncture has been shown to have anti-inflammatory effects, including regulation of proinflammatory cytokines, proinflammatory neuropeptides, and neurotrophins $[10,11]$. While CIAA is based on traditional acupuncture, persistent stimulus to acupoints by embedding catgut has been considered to extend its durative effect [12]. A systematic recent review on CIAA for AR indicated that CIAA is an effective therapy and has no adverse events [13]. Our previous studies have also confirmed the ameliorative 
effect of CIAA on AR symptoms in patients [14]. Previously, animal experiments also demonstrated that CIAA downregulated neurogenic inflammation, mainly induced by SP, CGRP (calcitonin gene-related peptide), and VIP (vasoactive intestinal peptide) [15].

AR is primarily an inflammatory IgE-mediated response [16], characterized by an enhanced Th2 immunological pattern (e.g., IL-4) and an inhibited Th1 pattern (such as IFN$\gamma$ ), together with the activation of mast cells, goblet cells, and eosinophils. Since excessive activation of Th2 drives AR, it is crucial to regulate the balance between Th1 and Th2 [17] to reverse the established Th2 response and avoid AR.

Toll-like receptors (TLRs) are natural immune receptors that recognize a variety of pathogen-associated molecular patterns (such as DAMPs or PAMPs) and play an important role in the immune defense response of the nasal mucosa $[18,19]$. TLRs are widely distributed in the $\mathrm{B}$ and Tlymphocytes, dendritic cells, and mast cells [20]. In addition to the PAMP, TLRs can further identify a large number of endogenous molecules derived from tissue damage [21], namely, stress cells, extracellular matrix degraded cytokines, and chemokines.

We have developed a rat model of ovalbumin- (OVA-) induced allergic inflammation, characterized by increased IL-4, sIgE and SP and reciprocal decrease of IFN- $\gamma$. In the present study, we have used this model to address the immunomodulatory effects of CIAA stimulation at Yingxiang (LI20) and Zusanli (ST36) acupoints and to elucidate the mechanisms involved in the regulation of SP, sIgE, IL-4, IFN- $\gamma$, TLR2, and TLR4. Our results provide a molecular foundation to the clinical applications of CIAA in treating AR.

\section{Materials and Methods}

2.1. Chemicals and Reagents. OVA (Sigma A8040, USA) was used as antigen, and aluminum hydroxide as adjuvant (lot No. 201110328, Chengdu Kelong Chemical Factory). The No. 9 needle (YZB/Su0313-2007, Yangzhou Co. Ltd., China), and the No. 000 catgut (YY1116-2002, Shanghai Co. Ltd., China) are disposable applications. sIgE, IL-4, and IFN- $\gamma$ enzymelinked immunosorbent assay (ELISA) kits were purchased from Abcam (Yonghui Bio Co. Ltd., Beijing, China). The SP and TLR2 polyclonal antibody (rabbit anti-mouse) and TLR4 monoclonal antibody (mouse anti-rat) were from Abcam (Trading Co. Ltd., Shang Hai, China). Sodium citrate buffer (0.01 M, pH 6.0) was prepared for dilution. Microscopic image acquisition and analysis system (MikeAudiBA200 Digital and Image-Pro Plus 6.0, USA) and ChemiDoc XRS gel imaging apparatus (Bio-Rad, USA) were purchased from the respective manufacturers.

2.2. Animal Preparation. Studies were carried out on adult male Sprague-Dawley rats (250-300g, 8-10 weeks of age), obtained from Da Shuo Biological Technology Co. Ltd., China, with certificate No. SCXK (Chengdu) 2015-030. Before the experiment began, all rats were adapted for 1 week in Experimental Animal Center of the Chengdu University of
TCM. In general, all procedures with animals avoided and / or minimized discomfort, distress, and pain to the animals. The rats were randomly divided into six groups $(n=7$ or 8 in each group): control, model, sham CIAA, CIAA for 1 week (C1), CIAA for 2 weeks (C2), and Budesonide-treated (by nasal spray).

2.3. Establishment of the AR Model. The AR model was established using an ovalbumin (OVA) sensitization method [15]. Rats were sensitized (days 1-13) with 7 intraperitoneal (i.p.) injections of $0.3 \mathrm{mg}$ OVA (Sigma A8040, USA) as antigen and $30 \mathrm{mg}$ aluminum hydroxide as adjuvant dissolved in $1 \mathrm{ml}$ of saline. Upon finishing the i.p. immunizations, the nasal antigen challenge (days 14-21) was performed with intranasal dripping of $50 \mu \mathrm{l}$ of $2 \%$ OVA daily for 7 consecutive days. The animals in the control group were administered with the same volume of saline. All animals were closely observed for developing any nasal responses of sneezing, watery rhinorrhea, and scraping for 30 minutes after each challenge. Then the symptoms and signs of AR were provoked (days 22-24) with intranasal dripping of $80 \mu \mathrm{l}$ of $1 \%$ OVA daily for 3 consecutive days. Ten minutes after the last provocation with $1 \%$ OVA, all animals were subjected to i.p. injection of $1 \%$ sodium pentobarbital $(50 \mathrm{mg} / \mathrm{kg})$ to collect tail venous blood. Serum levels of cytokines were measured using enzymelinked immunosorbent assay (ELISA). Specific IgE (sIgE), IL-4, and IFN- $\gamma$ levels were measured to test whether AR model was successfully established. Finally, the sensitization was maintained (day 25-end) with intranasal dripping of $50 \mu \mathrm{l}$ of $1 \%$ OVA every other day. At day 40 , final blood samples and nasal mucosa were collected for the various assays.

2.4. CIAA Treatment. The rats were placed in tailor-made mouse cages, so that their head and bilateral legs were sufficiently exposed. Stainless steel needles (No. 9) were bilaterally inserted at acupoint Yingxiang (LI20), 2-4 mm in depth, located at $\sim 3 \mathrm{~mm}$ on both sides of the nostril, and the catgut was pushed quickly into acupoint in the $\mathrm{Cl}$ and C2 group. At the same time, needles were bilaterally inserted at acupoint Zusanli (ST36), 2-4 $\mathrm{mm}$ in depth, located at 5 $\mathrm{mm}$ lateral and distal to the anterior tubercle of the tibia, and, as before, the catgut was pushed quickly into acupoint in the $\mathrm{C} 1$ and $\mathrm{C} 2$ group. All rats were conscious when CIAA was performed. In contrast to the CIAA groups, the sham group included sole acupuncture at point Yingxiang (LI20) and Zusanli (ST36) in the absence of catgut implantation. CIAA was conducted under sterile conditions. The tissue area was disinfected at the points with iodophor. Rats in the Budesonide group were intranasally administered with Budesonide ( $2.5 \mu \mathrm{g} /$ nasal cavity) daily for 14 consecutive days.

2.5. Assay of Rat Behavior. The numbers of sneezing and nose rubbing motions during $30 \mathrm{~min}$ after the final allergen challenge were recorded in each experimental group. Following superimposition of the recording results, a total score of $>5$ was used as benchmark for successful establishment of the AR [22]. 
2.6. Specimen Collection. The animals of all groups $(\mathrm{n}=$ 7 or 8 ) were sacrificed with i.p. injection of $3 \%$ sodium pentobarbital $(30 \mathrm{mg} / \mathrm{kg})$ and transcardially perfused with $350 \mathrm{~mL} 0.9 \%$ saline and fixed in a solution containing $2 \%$ paraformaldehyde and $1.25 \%$ glutaraldehyde phosphate buffer solution $(\mathrm{pH}=7.2)$. Blood collected from the femoral artery was subjected to measurements of serum sIgE, IL-4, and INF- $\gamma$. Part of the nasal mucosa was quickly removed from the respiratory area of the nasal chamber and postfixed in $4 \%$ paraformaldehyde, dissolved in $0.1 \mathrm{M}$ phosphate buffer. Another part of nasal mucosa was quickly frozen in liquid nitrogen. The tissues were sent for routine histological examination.

2.7. Degranulation Rate Assay of Mast Cells. Following the manufacturer's recommendations (toluidine blue, Biotech, USA), the degranulation rate of mast cells was detected by $1 \%$ toluidine blue staining, in which a total of 3 images for each sample were acquired and the number of degranulation of mast cells were recorded by microscopic examination. The degranulation rate (\%) of mast cells was then calculated.

2.8. Enzyme-Linked Immunosorbent Assay (ELISA). Serum levels of specific IgE (sIgE), IL-4, and IFN- $\gamma$ were measured by solid-phase enzyme-linked immunosorbent assay (ELISA) in accordance with the manufacturer's instructions. Bound immunoglobulin isotypes were detected with specific secondary antibody. Biotin-conjugated rat anti-mouse IgE, IL-4, and IFN- $\gamma$ antibodies were purchased from BD Pharmingen, Beijing, China.

2.9. Immunohistochemistry (IHC). Paraffin sections of nasal mucosa tissue were stained with streptavidin-peroxidase method to examine SP expression. The sections were incubated in 3\% hydrogen peroxide $\left(\mathrm{H}_{2} \mathrm{O}_{2}\right) /$ methanol for 15 min. After washing three times in PBS (pH 7.2-7.4) for 5 min each, they were immersed in $0.01 \mathrm{M}$ citrate buffer $(\mathrm{pH}$ 6.0) $5 \mathrm{~min}$, followed by two washes with PBS. Nonspecific binding was blocked by incubating with normal goat serum for $20 \mathrm{~min}$ at $37^{\circ} \mathrm{C}$. The sections were then incubated with rabbit anti-SP (1:200 dilution) overnight at $4^{\circ} \mathrm{C}$ and then with a biotinylated goat anti-rabbit IgG for $30 \mathrm{~min}$. Following incubation with horseradish peroxidase- (HRP) conjugated streptomycin ovalbumin reagent, the sections were colored using a concentrated DAB kit, and observation and acquisition of images were done in the mounting media. The immunopositive cells were detected using microscopic analysis (at 400x magnification), and the intensity of SPpositive expression was quantified by the average number of positively stained cells per field.

2.10. Immunofluorescence Analysis. The tissues, embedded in OCT and frozen in liquid nitrogen, were cut into serial 8 $\mu \mathrm{m}$ sections using a cryostat (Leica CM 1900, Bensheim, Germany), placed on APES (3-aminopropyltriethoxysilane)coated glass slides, dried at room temperature for $30 \mathrm{~min}$ and then stored in $-80^{\circ} \mathrm{C}[23,24]$. Sections were blocked with $1 \%$ fetal bovine serum (dissolved in PBS, supplemented with
$0.3 \%$ Triton $\mathrm{X}-100$ ) for $1 \mathrm{~h}$ at room temperature. They were then incubated with antibodies against TLR2 and TLR4 in a humid chamber at $4^{\circ} \mathrm{C}$ overnight. After rinsing with $0.1 \mathrm{M}$ PBS three times, the sections were incubated with secondary fluorescein-conjugated antibodies for $60 \mathrm{~min}$ at $37^{\circ} \mathrm{C}$ and then with DAPI (Beyotime $5 \mathrm{mg} / \mathrm{ml}$, China) to stain the nuclei for $30 \mathrm{~min}$ at room temperature. Images were acquired using a confocal microscope (A1R; Nikon, Japan).

2.11. Immunoblot (Western Blot). Cryopreserved tissues were thawed in a $37^{\circ} \mathrm{C}$ water bath and lysed by the addition of $2.5 \mathrm{~mL}$ of RIPA lysate (RIPA : cocktail = $100: 1$ ). Lysates were centrifuged at $12,000 \mathrm{rpm}$ for $30 \mathrm{~min}$ at $4^{\circ} \mathrm{C}$. The protein concentration of the cytosolic fraction was determined using a bicinchoninic acid (BCA) protein assay kit. The samples were boiled at $100^{\circ} \mathrm{C}$ in a sodium dodecyl sulfate (SDS) gel loading buffer for $10 \mathrm{~min}$ and loaded onto a 10\% SDS polyacrylamide gel. The separated proteins were electrotransferred to a membrane, which were then incubated in 5\% skim milk for $60 \mathrm{~min}$ at room temperature to block nonspecific binding. They were then incubated with rabbit antibodies against TLR2 (1:1000 dilution) or TLR4 (1:1000 dilution) overnight at $4^{\circ} \mathrm{C}$. The membranes were also probed with a monoclonal antibody specific for actin (1:5000 dilution, MAB1501, Chemicon) as an internal control for the cytosolic fraction. After washing, membranes were incubated with HRP-linked anti-rabbit IgG (1:5000 dilution) and HRP-linked anti-mouse IgG (1:5000 dilution) antibody in PBS for $1 \mathrm{~h}$ at RT. Proteins were detected using an enhanced chemiluminescence reagent kit. Densitometric analysis was performed using Quantity One software package. The TLR2/ $\beta$-actin and TLR $4 / \beta$-actin grayscale signal ratios were quantified.

2.12. Statistical Analysis. Data are presented as mean \pm standard deviation (SD) (SPSS Statistical analysis software version 20.0). All variables indicated approximately normal distribution by Kolmogorov-Smirnov test and homogeneity by Levene's test, simultaneously one-way analysis of variance (ANOVA), followed by post hoc analysis using the StudentNewman-Keuls- (SNK-) q test. Differences were considered to be statistically significant at a $\mathrm{P}$ value $<0.05(P<0.05)$.

\section{Results}

3.1. Establishment of the AR Model. In order to evaluate whether the AR model was established in the animal, tail venous blood from 7-8 rats, belonging to model, sham, $\mathrm{Cl}$, $\mathrm{C} 2$, and Budesonide groups, were collected. Serum-specific $\operatorname{IgE}$ (sIgE), IL-4, and IFN- $\gamma$ levels, compared to the control group, were used for assessment of AR. While the sIgE levels are strong diagnostic indicators, the levels of IL- 4 and IFN- $\gamma$, respectively, reflect the Th2 and Th1 cell populations. Results are presented as mean $\pm \mathrm{SD}$, and $P<0.05$ or $P<0.01$ was considered statistically significant differences (Table 1 ).

In what follows, we have used this animal model to test the effects of CIAA on multiple biological and molecular parameters, relevant to allergic rhinitis (AR). 
TABLE 1: Serum levels of sIgE, IFN- $\gamma$, and IL-4 (mean \pm SD).

\begin{tabular}{lccc}
\hline Group & $\operatorname{sIgE}(\mathrm{ng} / \mathrm{mL})$ & $\mathrm{IFN}-\gamma(\mathrm{mg} / \mathrm{mL})$ & $\mathrm{IL}-4(\mathrm{pg} / \mathrm{mL})$ \\
\hline Control & $72.65 \pm 9.17$ & $0.59 \pm 0.06$ & $10.74 \pm 3.05$ \\
Model & $124.92 \pm 15.56^{\#}$ & $0.29 \pm 0.07^{\triangle}$ & $16.04 \pm 3.81 *$ \\
Budesonide & $126.34 \pm 13.20^{\#}$ & $0.27 \pm 0.05^{\triangle}$ & $16.75 \pm 5.01 *$ \\
Sham & $127.06 \pm 14.20^{\#}$ & $0.26 \pm 0.06^{\triangle}$ & $16.90 \pm 2.75 *$ \\
C1 & $129.20 \pm 11.12^{\#}$ & $0.28 \pm 0.06^{\triangle}$ & $17.32 \pm 3.05 *$ \\
C2 & $127.49 \pm 12.51^{\#}$ & $0.25 \pm 0.05^{\triangle}$ & $17.18 \pm 2.98 *$ \\
\hline
\end{tabular}

Note: serum levels were measured as described under Methods. $* P<0.01$ versus the control group; ${ }^{\wedge} P<0.01$ versus the control group; ${ }^{\#} P<0.01$ versus the control group.

\subsection{Effects of CIAA on Animal Behavior. Behavior evaluation} of each group of rat included studies of sneezing, scratching, nasal discharge, and foraging symptoms. The behavior scores showed no apparent difference between the model group and sham group $(P>0.05)$. However, values of the $\mathrm{C} 2$ group were significantly lower than those of the model, sham and $\mathrm{Cl}$ groups $(P<0.01)$. We also observed that the Budesonide group showed no significant difference compared to the $\mathrm{C} 2$ group $(P$ $>0.05$, Figure 1).

3.3. Effects of CIAA on the Degranulation Rates of Mast Cells. The degranulation rates of mast cells were examined by microscopy. A total of 3 images for each sample were acquired and the number of degranulation of mast cells was counted and recorded. The degranulation rates of the mast cells showed no significant difference among the C1,C2 and Budesonide groups $(P>0.05)$. However, the rates in the $\mathrm{C} 2$ and Budesonide groups were significantly lower than the model or sham groups $(P<0.01)$. The $\mathrm{C} 1$ group also showed a lower rate, compared to the model or sham group $(P<0.05$; Figure 2).

3.4. Effects of CIAA on sIgE, IL-4, and IFN- $\gamma$ Levels. In ELISA analysis (Figure 3 ), the serum $\operatorname{IgE}(\operatorname{sgE})$ showed no significant difference among the $\mathrm{C} 1, \mathrm{C} 2$, and Budesonide groups $(\mathrm{P}>0.05)$. However, the sIgE was significantly lower in the $\mathrm{C} 2$ and Budesonide groups than that in the model and sham groups $(\mathrm{P}<0.01$; Figure $3(\mathrm{a}))$. Besides, the model group was similar to the sham group in sIgE levels. The serum IL4 showed no significant difference among the $\mathrm{C} 1, \mathrm{C} 2$, and Budesonide groups $(\mathrm{P}>0.05)$. However, the serum IL-4 was significantly lower in the $\mathrm{C} 2$ and Budesonide groups than in the model and sham groups $(\mathrm{P}<0.01$; Figure $3(\mathrm{~b}))$, but the model and the sham groups were similar $(\mathrm{P}>0.05)$. Lastly, the serum IFN- $\gamma$ showed no significant difference between the $\mathrm{C} 1, \mathrm{C} 2$, and Budesonide groups $(\mathrm{P}>0.05)$. However, it was lower in the Budesonide group than in the $\mathrm{Cl}$, model, and sham groups $(P<0.05$ and $P<0.01$, Figure $3(\mathrm{c}))$, whereas the model and the sham groups were similar with no significant difference $(P>0.05)$.

3.5. Effect of CIAA on SP Expression. In the immunohistochemical (IHC) analysis (Figure 4), the expression of SP in the model group showed significant difference from the $\mathrm{C} 1$, $\mathrm{C} 2$, and Budesonide groups $(P<0.01)$. The expression was

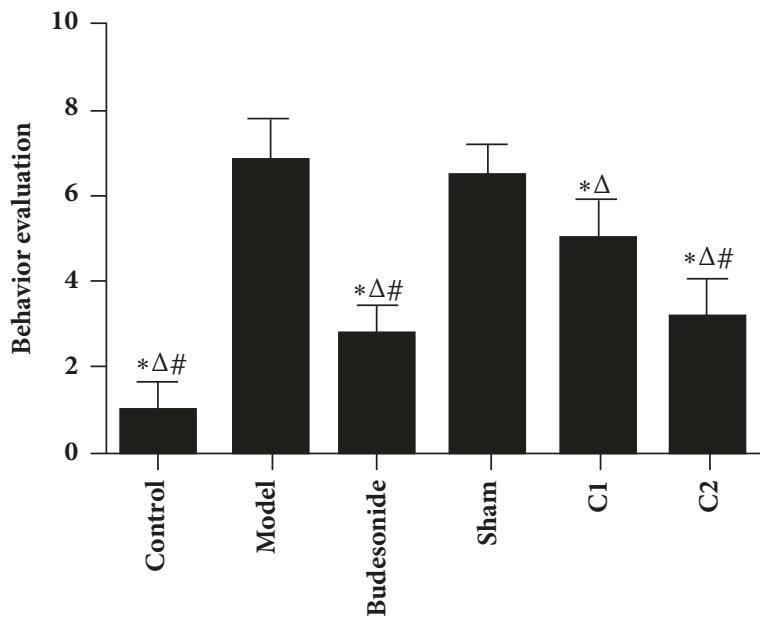

FIGURE 1: Effect of CIAA on behavior evaluation. The experimental protocol, including the administration of CIAA in rats, the various controls, and measurement of animal behavior have been detailed in Materials and Methods. $* P<0.01$ versus model group; ${ }^{\triangle} P<0.01$ versus sham group; and ${ }^{\#} P<0.01$ versus $\mathrm{Cl}$ group.

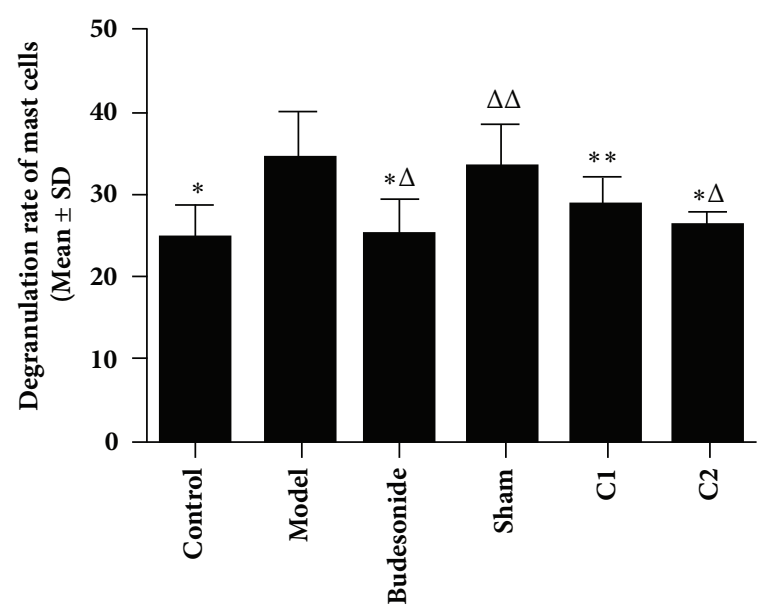

FIGURE 2: Effects of CIAA on the degranulation rates of mast cells. The experimental protocol (essentially the same as in Figure 1) and measurement of degranulation rates have been detailed in Materials and Methods. $* P<0.01$ versus the model group; $* * P<0.05$ versus the model group; ${ }^{\triangle} P<0.01$ versus the sham group; ${ }^{\triangle} P<0.05$ versus the $\mathrm{C} 1$ group; $\mathrm{C} 1$ versus $\mathrm{C} 2 \mathrm{P}>0.05$, Budesonide versus $\mathrm{C} 1 / \mathrm{C} 2, P>$ 0.05 . 


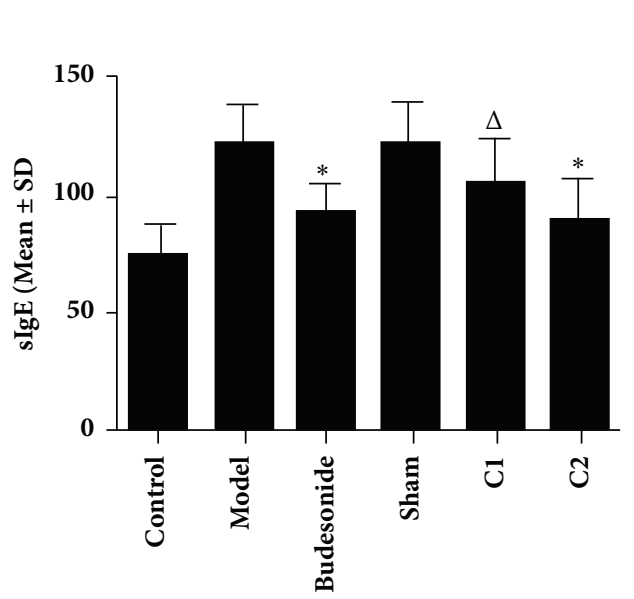

(a)

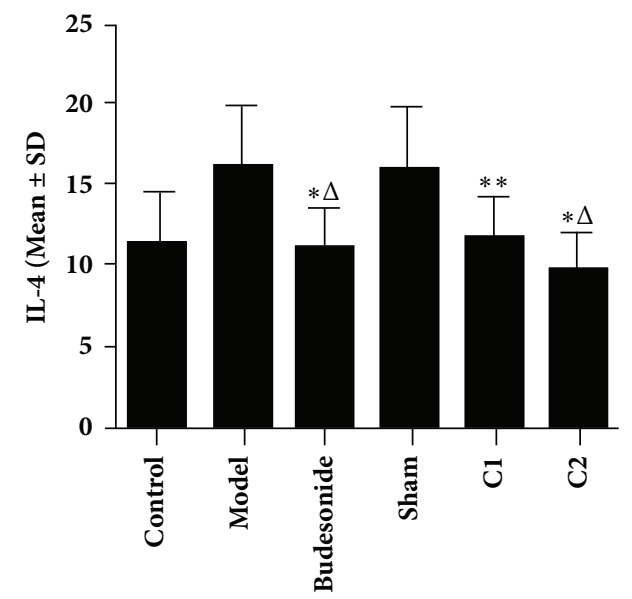

(b)

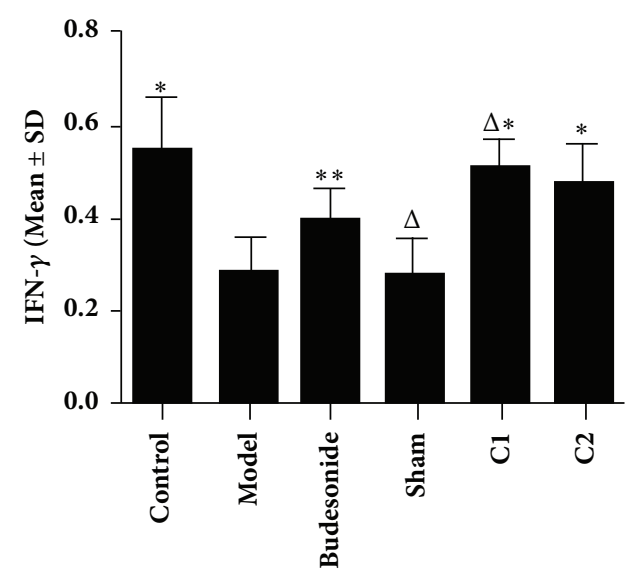

(c)

FIgURE 3: Effects of CIAA on (a) sIgE, (b) IL-4, and (c) IFN- $\gamma$ levels. The experimental protocol (essentially the same as in Figure 1) and measurement of the three molecules have been detailed in Materials and Methods. (a) sIgE: $* P<0.01$ versus the model group; ${ }^{\triangle} P<0.05$ versus the model group; $\mathrm{C} 1$ versus $\mathrm{C} 2 P>0.05$; Budesonide versus $\mathrm{C} 1 / \mathrm{C} 2, P>0.05$. (b) IL-4: $* P<0.01$ versus the model group; $* * P<0.05$ versus the model group; ${ }^{\triangle} P<0.01$ versus the sham group; $\mathrm{C} 1$ versus $\mathrm{C} 2, P>0.05$; Budesonide versus $\mathrm{C} 1 / \mathrm{C} 2, P>0.05$; (c) IFN- $\gamma: * P<0.01$ versus the model group; $* * P<0.05$ versus the model group; ${ }^{\triangle} P<0.05$ versus the Budesonide group; $\mathrm{C} 1$ versus $\mathrm{C} 2, P>0.05$; Budesonide versus $\mathrm{C} 2, P>0.05$.

lower in the Budesonide group than in the $\mathrm{Cl}$ groups $(P<$ 0.05 ; Figures $4(\mathrm{a})$ and $4(\mathrm{~b})$ ), whereas the $\mathrm{C} 2$ group showed no significant difference with the Budesonide group $(P>0.05)$. The expression in the $\mathrm{C} 2$ group was slightly lower than that in $\mathrm{Cl}$.

3.6. Effects of CIAA on TLR2 and TLR4 Expression. In our immunofluorescence analysis (Figure 5), we could not detect any significant difference in the expression of TLR2 in the nasal mucosa among the $\mathrm{C} 1, \mathrm{C} 2$, model, and sham groups $(P>0.05)$. However, the TLR2 expression was lower in the Budesonide group than in the model, sham, and $\mathrm{C} 1$ groups $(P<0.05)$. We also did not observe any significant difference in the expression of TLR2 in the Budesonide group, compared with the $\mathrm{C} 2$ group $(P>0.05)$. TLR4 expression in the nasal mucosa also showed no significant difference among the $\mathrm{C} 1, \mathrm{C} 2$, and Budesonide groups $(P>0.05)$ but was significantly lower in the Budesonide group compared to model and sham groups $(P<0.01$; Figures 5(a)-5(c)). TLR4 expression was also lower in the $\mathrm{C} 1$ and $\mathrm{C} 2$ groups than in the model and sham groups $(P<0.05)$. Lastly, TLR4 in the model group showed no significant difference compared with the sham group $(P>$ 0.05).

3.7. Effects of CIAA on the Cytosolic Expression of TLR2 and TLR4. In Western blot analysis of fractionated cell extracts, we detected the cytosolic expression of TLR2 and TLR4 in the nasal mucosal cells, which showed no significant difference among the $\mathrm{C} 1, \mathrm{C} 2$, and Budesonide groups $(P>0.05)$, but both were significantly lower in the $\mathrm{C} 1, \mathrm{C} 2$, and Budesonide groups than in the model or sham group $(P<0.01$; Figures $6(a)-6(c))$. Furthermore, the expression of TLR2 and TLR4 in the sham group showed no significant difference compared with the model group $(P>0.05)$. 


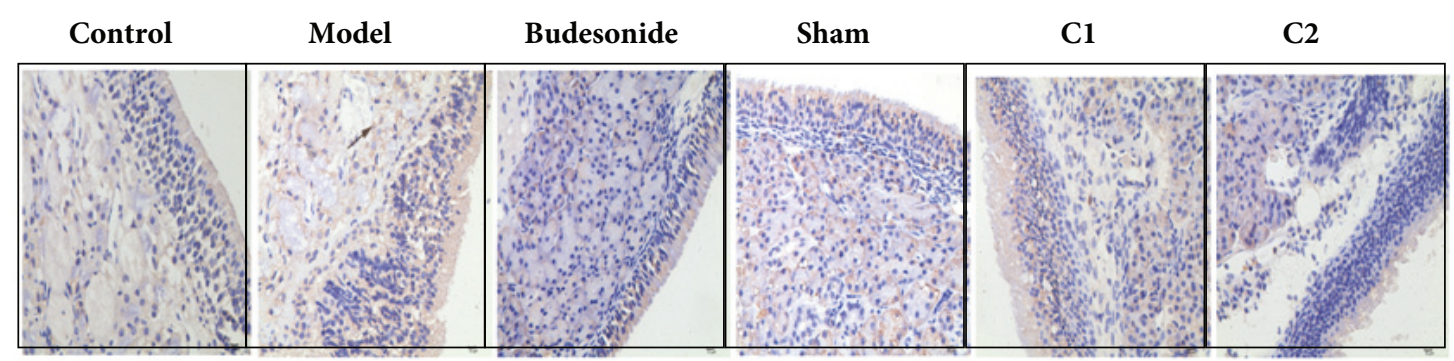

(a)

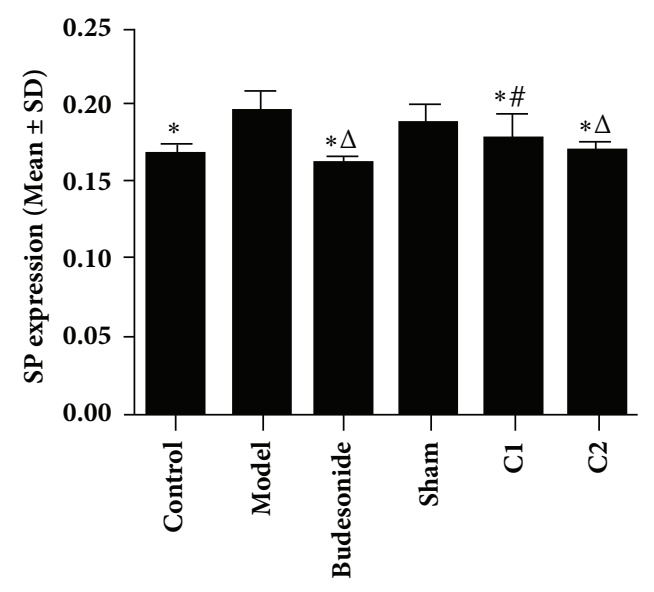

(b)

FIGURE 4: Effects of CIAA on SP expression. The experimental protocol (essentially the same as in Figure 1) and measurement of SP expression have been detailed in Materials and Methods. (a) IHC image; (b) quantification. $* P<0.01$ versus the model group. ${ }^{\triangle} P<0.01$ versus the sham group. ${ }^{\#} P<0.05$ versus the $\mathrm{Cl}$ group. Scale bar for panel $(\mathrm{a})=50 \mu \mathrm{m}$.

\section{Discussion}

CIAA is based on the theory and practice of traditional acupuncture, which can extend the sensation and effect of needling because of the persistent stimulus to acupoints by the embedding catgut $[14,25]$. Our results, presented here, reveal that CIAA has significant inhibitory effects on allergic inflammation, especially in inhibiting nasal symptoms and the degranulation rates of mast cells in nasal mucosa and the related proinflammatory cytokines, which is consistent with previous studies of ours and others on $\operatorname{AR}[25,26]$. Our results clearly showed that the Th2 cytokine (IL-4) and sIgE levels significantly decreased after CIAA treatment; in contrast, the Thl cytokine (IFN- $\gamma$ ) significantly increased, which is in agreement with recent evidence of significant increase in IFN- $\gamma$ and decrease in IL-4 $[27,28]$. In addition, we found significant decrease of SP expression following CIAA treatment, which is also in agreement with several published studies $[11,15,29]$. Finally, in conditions of nasal hypersensitivity and compromised mucosal integrity, the expression levels of TLR2 and TLR4 significantly increased in the model group. However, CIAA is considered an activating stimulus to the tissue, mainly caused by needle injury and catgut homologous protein, which significantly downregulated TLR2 and TLR4 expression.
In China, CIAA has been particularly effective in treating chronic diseases and used for centuries to treat AR. While the majority of studies has focused on the anti-inflammatory mechanisms of acupuncture research, few have investigated the immunomodulatory effects of CIAA obtained from rats. In this study, we addressed the immunomodulatory effects of CIAA on AR, providing a basis for further clinical applications of CIAA on treating allergic diseases. In addition, our results demonstrate a potentially beneficial regimen in the treatment of AR.

The exact pathological mechanism of AR is currently not fully understood. Accumulating evidence has shown that neuroimmune abnormalities play important roles in the development of AR [30]. Excessive activation of T-helper 2 (Th2) and mast cell-mediated inflammatory reactions play a central role in AR. Specifically, several studies have shown that the Th2 cytokines, such as IL-4, are downregulated by CIAA or acupuncture $[31,32]$ and that the Th1 cytokines, such as IFN- $\gamma$, are also regulated [31, 33, 34]. Yet another study reported a reduction of IgE concentrations in the blood [35]; in this study, IL-4 levels significantly decreased after weeks of CIAA, and so did sIgE levels, while IFN- $\gamma$ levels were significantly upregulated. These results support the proposal that a shift from Th2 to Th1 occurs after CIAA treatment, especially around two weeks. 

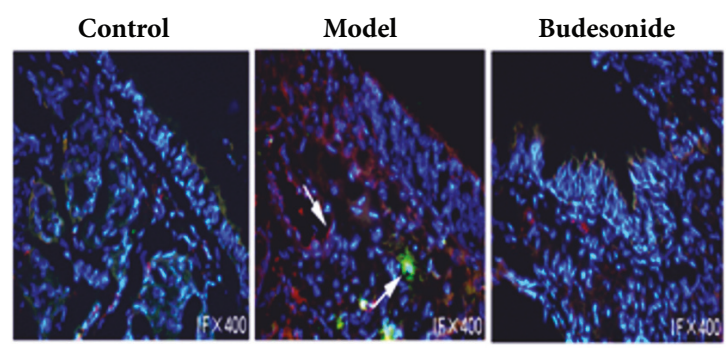

Sham

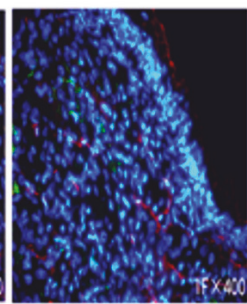

C1

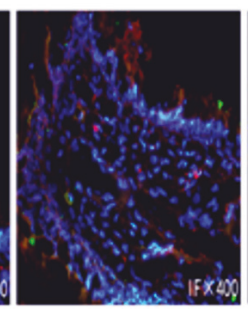

$\mathrm{C} 2$

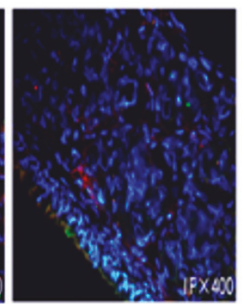

(a)

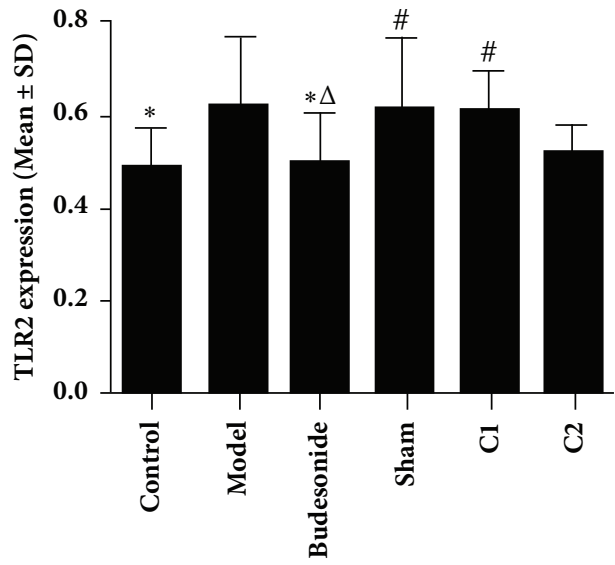

(b)

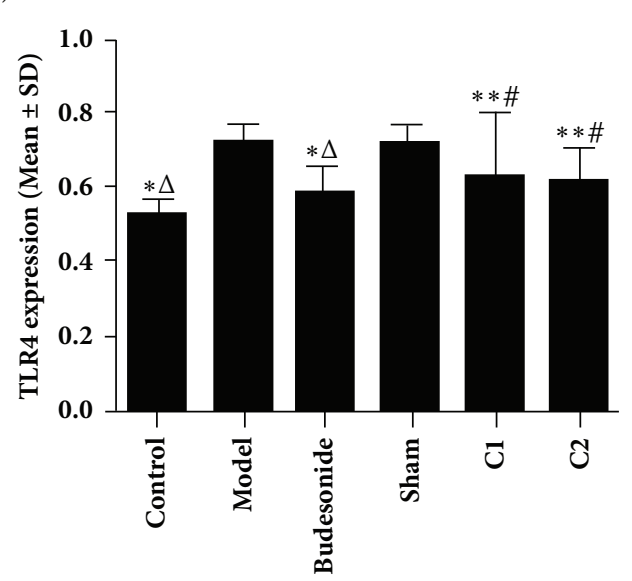

(c)

FIGURE 5: Effects of CIAA on TLR2 and TLR4 expression. The experimental protocol including fluorescence assays has been described in Materials and Methods. (a) Representative photographs showing TLR2 (green) and TLR4 (red) are shown. Nuclei were stained with DAPI (blue). The data are presented as mean $\pm \mathrm{SD}$. (b) TLR2 quantification: $* \mathrm{P}<0.05$ versus the model group; ${ }^{\triangle} \mathrm{P}<0.05$ versus the sham group; ${ }^{\#} \mathrm{P}<0.05$ versus Budesonide group. (c) TLR4 quantification: $* \mathrm{P}<0.01$ versus the model group; $* * \mathrm{P}<0.05$ versus the model group; ${ }^{\triangle} \mathrm{P}<0.01$ versus the sham group; ${ }^{*} \mathrm{P}<0.05$ versus the sham group.

In addition to the above-mentioned immune cell activation, neurogenic inflammation, such as SP expression in nasal mucosa, decreased after CIAA, which is in accordance with previous studies [11]. Our results (Figure 4) showed a trend in which the C2 group is lower than the C1 group in SP expression. This apparent effect of CIAA can be attributed to the persistent stimulus to acupoints by the embedding catgut. It is now well established that substance $\mathrm{P}$ plays an essential role in neurogenic inflammation in the nasal mucosa $[36,37]$. SP is released from sensory nerves via axonal reflex, leading to vasodilation and plasma exudation to promote AR symptoms. However, the early-phase allergic response is mainly caused by the chemical mediators secreted from mast cells and basophils. Moreover, the chemical mediators stimulate nasal sensory neurons to release many neuropeptides, such as SP; histamine, a strong neurotransmitter, is also released in allergic inflammatory processes [38-41]. Overall, an interactive role between cytokines and neuropeptides underlies such allergic responses. Together, these results lead us to conclude that CIAA attenuates the development of nasal neurogenic and cytokine-mediated inflammation and eventually inhibits AR development.

Additionally, expression of Toll-like receptors, TLR2 and TLR4, which are upstream effectors of signaling pathways, decreased after CIAA. Our trend shows that the C2 group was lower than $\mathrm{C} 1$ in TLR2 and TLR4 expression, likely associated with the persistent stimulus of embedding catgut. A number of studies have identified associations between Toll-like receptor genes and AR [42]. In particular, TLR2 and TLR4, which are expressed on the surfaces of diverse immune cells, including dendritic cells, macrophages, and B- and T-lymphocytes [43], can modify cellular immune response and alter the host's susceptibility to disease [44].

There were some limitations associated with the current study, in part because all possible ranges of variables were not investigated. First, we did not examine other neuropeptides such as CGRP and VIP. Second, the exact mechanisms underlying the observed improvement in the model of AR through CIAA administration are likely to be more complex and remain to be elucidated. Third, although the C2 group showed the tendency to be lower than $\mathrm{C} 1$ in inflammatory readouts, the difference is not remarkable and its significance is unclear. Perhaps, if we provide a second CIAA on the rats, the effects of CIAA may be different from the present study. Future studies, incorporating more extensive experimental designs, complemented by in vitro studies, should provide a mechanistic basis for improvement of AR by CIAA treatment. 


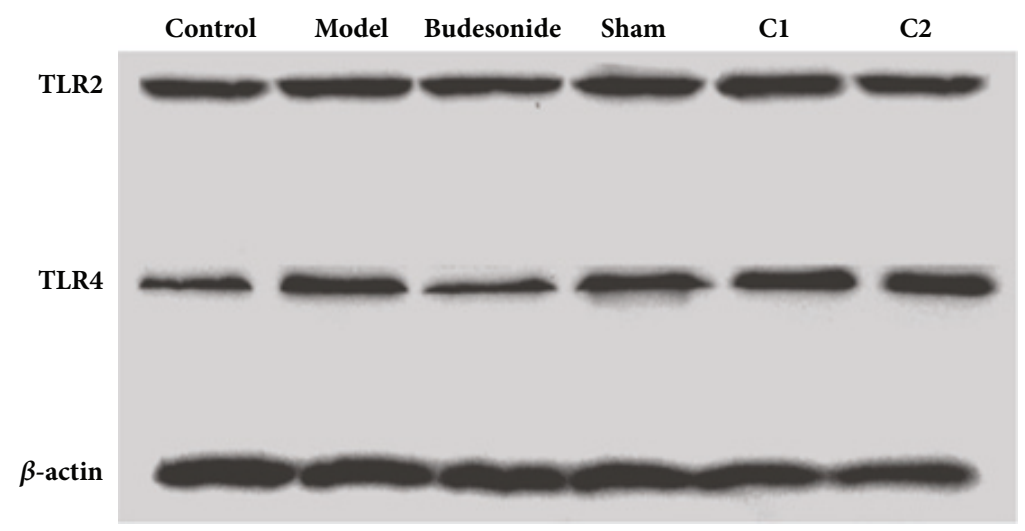

(a)

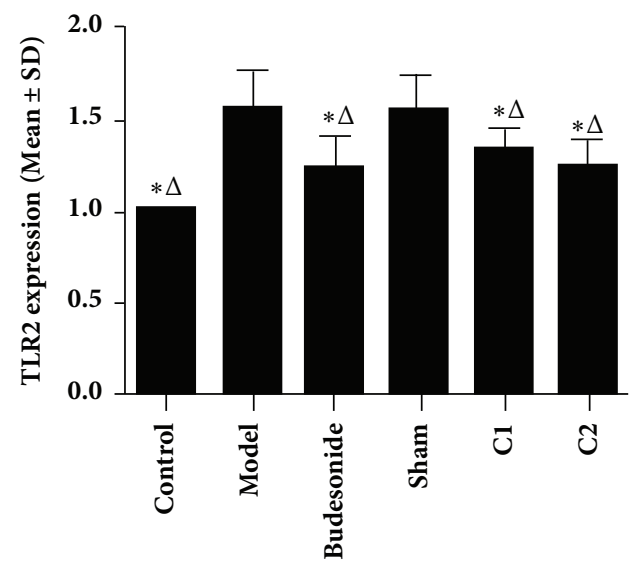

(b)

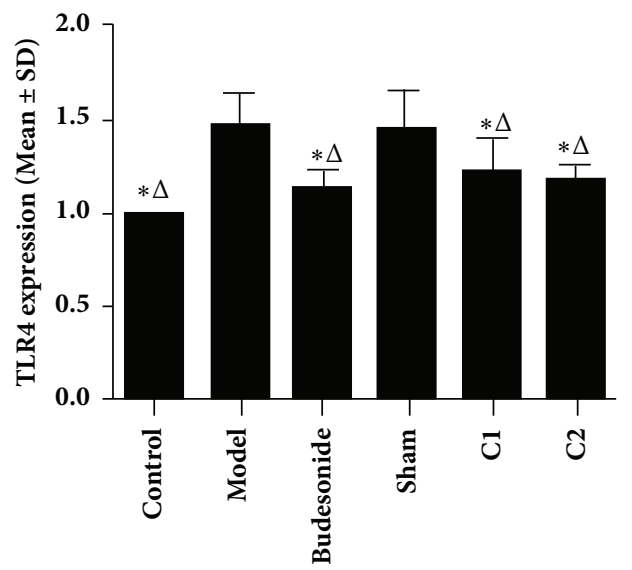

(c)

FIgURE 6: Effects of CIAA on the cytosolic expression of TLR2 and TLR4. (a) Representative Western blot images showing cytosolic expression of TLR2 and TLR4 in the indicated groups, as described in Materials and Methods. Actin serves as an internal control. (b) TLR2: $* P<0.01$ versus the model group; ${ }^{\triangle} P<0.01$ versus the sham group. (c) TLR4: $* P<0.01$ versus the model group; ${ }^{\triangle} P<0.01$ versus the sham group.

\section{Conclusion}

Based on the collective results, we conclude that CIAA at the Yingxiang (LI20) and Zusanli (ST36) acupoints effectively reduced allergic symptoms and inflammatory parameters in the rat model of AR. We suggest that CIAA treatment is potentially an alternative therapeutic modality in AR.

\section{Data Availability}

Please contact author for any additional data requests.

\section{Ethical Approval}

There were no human subjects. The institutional committee of the Chengdu University of TCM certified that the proposed experimental procedures complied with appropriate ethics and the Animal Protection Law.

\section{Conflicts of Interest}

All authors declare that they have no conflicts of interest. The funders were not involved in the study design, data collection and analysis, decision to publish, or preparation of the manuscript.

\section{Authors' Contributions}

Qinxiu Zhang, Shasha Yang, Jing Wu, Xinrong Li, Daien Liu, Bin Zeng, Hongjiao Gao, and Xiaolin Yan conceived and designed the experiments. Shasha Yang and Jing $\mathrm{Wu}$ analyzed the data. Shasha Yang and Jing $\mathrm{Wu}$ wrote the manuscript. Qinxiu Zhang and Zhendong Zhong reviewed the manuscript. All authors approved the manuscript. Shasha Yang and Jing Wu contributed equally to this work.

\section{Acknowledgments}

This research was supported by the National Natural Science of China (no. 81273985 and no. 81473523) and by National Science \& Technology Pillar Program during the Twelfth Fiveyear Plan Period of China (no. 2015BAI04B00). The funders had no role in study design, data collection and analysis, decision to publish, or preparation of the manuscript. 


\section{References}

[1] S. R. Durham, S. Ying, V. A. Varney et al., "Cytokine messenger RNA expression for IL-3, IL-4, IL-5, and granulocyte/macrophage-colony-stimulating factor in the nasal mucosa after local allergen provocation: relationship to tissue eosinophilia," The Journal of Immunology, vol. 148, no. 8, pp. 2390-2394, 1992.

[2] D. I. Bernstein, G. Schwartz, and J. A. Bernstein, "Allergic Rhinitis: Mechanisms and Treatment," Immunology and Allergy Clinics of North America, vol. 36, no. 2, pp. 261-278, 2016.

[3] F. Horak and U. P. Zieglmayer, "Azelastine nasal spray for the treatment of allergic and nonallergic rhinitis," Expert Review of Clinical Immunology, vol. 5, no. 6, pp. 659-669, 2009.

[4] S. Fujieda, Y. Kurono, K. Okubo et al., "Examination, diagnosis and classification for Japanese allergic rhinitis: Japanese guideline," Auris Nasus Larynx, vol. 39, no. 6, pp. 553-556, 2012.

[5] A. Roger, E. Arcalá Campillo, M. C. Torres et al., "Reduced work/academic performance and quality of life in patients with allergic rhinitis and impact of allergen immunotherapy," Allergy, Asthma \& Clinical Immunology, vol. 12, no. 1, 2016.

[6] X. D. Wang, M. Zheng, H. F. Lou, C. S. Wang, Y. Zhang, and M. Y. Bo, "An increased prevalence of self-reported allergic rhinitis in major Chinese cities from 2005 to 2011," Allergy, vol. 71, p. 1170, 2016.

[7] Y. Wang, H. Chen, R. Zhu et al., "Allergic Rhinitis Control Test questionnaire-driven stepwise strategy to improve allergic rhinitis control: a prospective study," Allergy: European Journal of Allergy and Clinical Immunology, vol. 71, no. 11, pp. 1612-1619, 2016.

[8] C. L. Ng and D. Y. Wang, "Latest developments in allergic rhinitis in Allergy for clinicians and researchers," Allergy: European Journal of Allergy and Clinical Immunology, vol. 70, no. 12, pp. 1521-1530, 2015.

[9] M. D. Seidman, R. K. Gurgel, S. Y. Lin et al., "Clinical practice guideline: Allergic rhinitis executive summary," Otolaryngology - Head and Neck Surgery (United States), vol. 152, no. 2, pp. 197206, 2015.

[10] J. L. McDonald, A. W. Cripps, P. K. Smith, C. A. Smith, C. C. Xue, and B. Golianu, "The anti-inflammatory effects of acupuncture and their relevance to allergic rhinitis: a narrative review and proposed model," Evidence-Based Complementary and Alternative Medicine, vol. 2013, Article ID 591796, 12 pages, 2013.

[11] Y. M. Li, L. X. Zhuang, X. S. Lai, and G. H. Jiang, "Effects of electroacupuncture on plasma vasoactive intestinal peptide and substance $\mathrm{P}$ in perennial allergic rhinitis patients," Zhen Ci Yan Jiu, vol. 32, pp. 136-138, 2007.

[12] R. Yan, X. Liu, J. Bai, J. Yu, and J. Gu, "Influence of catgut implantation at acupoints on leptin and insulin resistance in simple obesity rats," Journal of Traditional Chinese Medicine, vol. 32, no. 3, pp. 477-481, 2012.

[13] X.-R. Li, Q.-X. Zhang, M. Liu et al., "Catgut Implantation at acupoints for allergic rhinitis: A systematic review," Chinese Journal of Integrative Medicine, vol. 20, no. 3, pp. 235-240, 2014.

[14] X. R. Li, Q. X. Zhang, and L. Y. Jiang, "Clinical effect of catgut implantation at acupoints for allergic rhinitis: study protocol for a randomized controlled trial," Trials, vol. 14, article 12, 2013.

[15] X. R. Li, Q. X. Zhang, X. P. Wang, Y. Liu, Q. Chen, and Z. D. Zhong, "Effects of catgut implantation at acupoint of face on regulating nasal mucosa neurogenic inflammation of rats with allergic rhinitis," Chin J of TCM and Pharmacy, vol. 29, pp. 25872590, 2014.

[16] S. Işık, M. Karaman, A. Adan et al., "Intraperitoneal mesenchymal stem cell administration ameliorates allergic rhinitis in the murine model," European Archives of Oto-Rhino-Laryngology, vol. 274, no. 1, pp. 197-207, 2017.

[17] N. Zhao, Y. Liu, H. Liang, and X. Jiang, "Bone marrow-derived mesenchymal stem cells reduce immune reaction in a mouse model of allergic rhinitis," Am J Transl Res, vol. 8, pp. 5628-5636, 2016.

[18] A. Dębińska and A. Boznański, "The role of Toll-like receptors in the pathogenesis of allergic diseases - where is the truth?" Postepy Higieny i Medycyny Doswiadczalnej, vol. 68, pp. 230237, 2014.

[19] Z. Dong, L. Xiong, W. Zhang et al., "Holding the inflammatory system in check: TLRs and their targeted therapy in asthma," Mediators of Inflammation, vol. 2016, Article ID 2180417, 8 pages, 2016.

[20] L. J. Vanhinsbergh, D. G. Powe, and N. S. Jones, "Reduction of TLR2 gene expression in allergic and nonallergic rhinitis," Annals of Allergy, Asthma \& Immunology, vol. 99, no. 6, pp. 509516, 2007.

[21] S. Akira, "Mammalian Toll-like receptors," Current Opinion in Immunology, vol. 15, no. 1, pp. 5-11, 2003.

[22] X. J. Zhao, "Experimental models of nasal hypersensitive reaction," Zhonghua Er Bi Yan Hou Ke Za Zhi, vol. 28, no. 17-18, pp. 58-59, 1993.

[23] S. Hong, J. Fan, E. S. Kemmerer, S. Evans, Y. Li, and J. W. Wiley, "Reciprocal changes in vanilloid (TRPV1) and endocannabinoid (CB1) receptors contribute to visceral hyperalgesia in the water avoidance stressed rat," Gut, vol. 58, no. 2, pp. 202-210, 2009.

[24] D. D. Le, S. Rochlitzer, A. Fischer et al., "Allergic airway inflammation induces the migration of dendritic cells into airway sensory ganglia," Respiratory Research, vol. 15, no. 1, article no. 73, 2014.

[25] M. Liu, D. Z. Zhang, X. R. Li, F. B. Zhang, X. P. Wang, and Y. H. Qi, "Effects of catgut implantation at acupoint of face on regulating nasal mucosa IFN- $\gamma$, IL- 4 of rats with allergic rhinitis," Jiangsu Journal of Traditional Chinese Medicine, vol. 48, pp. 78-80, 2016.

[26] J. Chen, X.-S. Lai, C.-Z. Tang, L.-L. He, and B.-X. Jin, "Effects of point application on celiac mast cell degranulation in mice with allergic rhinitis: An experimental study," Journal of Acupuncture and Tuina Science, vol. 8, no. 2, pp. 75-79, 2010.

[27] Y. Q. Rao and N. Y. Han, "Therapeutic effect of acupuncture on allergic rhinitis and its effects on immunologic function," Zhongguo Zhen Jiu, vol. 26, pp. 557-560, 2006.

[28] M.-F. Zheng, C. Lin, L.-P. Zheng, and F.-R. He, "Effects of acupuncture-moxibustion on monocyte Th1/Th2 cytokine in peripheral blood of patients with perennial allergic rhinitis," Journal of Acupuncture and Tuina Science, vol. 8, no. 2, pp. 8588, 2010.

[29] M. K. Ai, X. M. Guan, C. G. Zhu, and L. L. Vacca, "The influence of electroacupuncture and morphine on substance $\mathrm{P}$ (SP), enkephalin (ENK) and acetylcholine esterase (AChE) in the spinal cord of rats," Zhen Ci Yan Jiu, vol. 11, pp. 96-105, 1986.

[30] S. Sarin, B. Undem, A. Sanico, and A. Togias, "The role of the nervous system in rhinitis," The Journal of Allergy and Clinical Immunology, vol. 118, no. 5, pp. 999-1014, 2006. 
[31] M. Liu, D. Z. Zhang, X. R. Li, F. B. Zhang, and X. P. Wang, "Catgut implantation at acupoint regulating immune microenvironment of nasal mucosa in allergic rhinitis rats," Journal of Shandong University of Ear, Eye, Nose, and Throat, vol. 30, pp. 9-13, 2016.

[32] Y. Q. Rao and N. Y. Han, "Clinical effects and influence on serum immunology of acupuncture for allergic rhinitis," Chinese Acupuncture and moxibustion, vol. 26, pp. 557-560, 2006.

[33] B. Hauswald and Y. M. Yarin, "Acupuncture in allergic rhinitis," Allergo Journal International, vol. 23, no. 4, pp. 115-119, 2014.

[34] E. R. Carneiro, R. A. N. Xavier, M. A. P. D. Castro, C. M. O. D. Nascimento, and V. L. F. Silveira, "Electroacupuncture promotes a decrease in inflammatory response associated with Th1/Th2 cytokines, nitric oxide and leukotriene B4 modulation in experimental asthma," Cytokine, vol. 50, no. 3, pp. 335-340, 2010.

[35] C. Jianli, "The effect of acupuncture on serum IgE level in patients with chronic urticaria," Journal of Traditional Chinese Medicine, vol. 26, pp. 189-190, 2006 (Chinese).

[36] I. Ruocco, A. C. Cuello, R. Shigemoto, and A. Ribeiro-da-Silva, "Light and electron microscopic study of the distribution of substance P-immunoreactive fibers and neurokinin-1 receptors in the skin of the rat lower lip," Journal of Comparative Neurology, vol. 432, no. 4, pp. 466-480, 2001.

[37] M. Kashiwabara, K. Asano, T. Mizuyoshi, and H. Kobayashi, "Suppression of neuropeptide production by quercetin in allergic rhinitis model rats," BMC Complementary and Alternative Medicine, vol. 16, no. 1, article no. 132, 2016.

[38] G. Hanf, K. Schierhorn, T. Brunnée, O. Noga, D. Verges, and G. Kunkel, "Substance P induced histamine release from nasal mucosa of subjects with and without allergic rhinitis," Inflammation Research, vol. 49, no. 10, pp. 520-523, 2000.

[39] B. P. Tancowny, V. Karpov, R. P. Schleimer, and M. Kulka, "Substance P primes lipoteichoic acid- and Pam3CysSerLys4mediated activation of human mast cells by up-regulating Tolllike receptor 2," The Journal of Immunology, vol. 131, no. 2, pp. 220-230, 2010.

[40] N. Gaudenzio, R. Sibilano, T. Marichal et al., "Different activation signals induce distinct mast cell degranulation strategies," The Journal of Clinical Investigation, vol. 126, no. 10, pp. 39813998, 2016.

[41] H. Nishijima, K. Kondo, M. Toma-Hirano et al., "Denervation of nasal mucosa induced by posterior nasal neurectomy suppresses nasal secretion, not hypersensitivity, in an allergic rhinitis rat model," Laboratory Investigation, vol. 96, no. 9, pp. 981-993, 2016.

[42] D. Nilsson, A. K. Andiappan, C. Halldén et al., “Toll-like receptor gene polymorphisms are associated with allergic rhinitis: a case control study," BMC Medical Genetics, vol. 13, article no. 66, 2012.

[43] L. Zuo, K. Lucas, C. A. Fortuna, C. Chuang, and T. M. Best, "Molecular regulation of toll-like receptors in asthma and COPD," Frontiers in Physiology, vol. 6, p. 312, 2015.

[44] E. A. Misch and T. R. Hawn, "Toll-like receptor polymorphisms and susceptibility to human disease," Clinical Science, vol. 114, no. 5-6, pp. 347-360, 2008. 


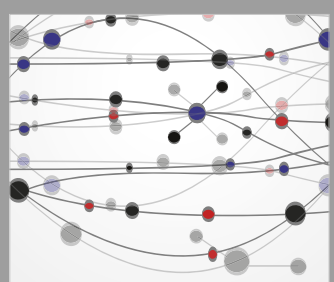

The Scientific World Journal
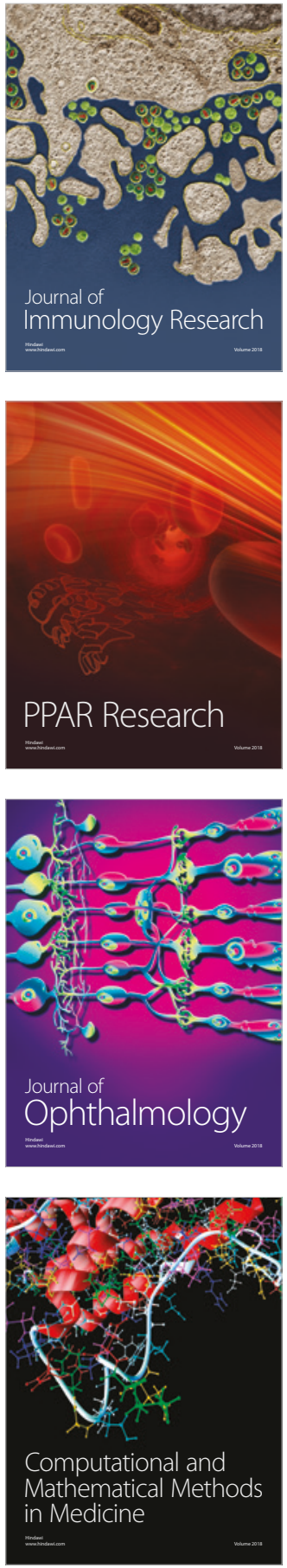

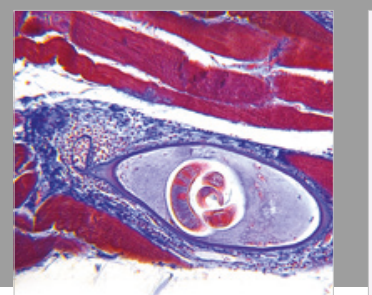

Gastroenterology Research and Practice

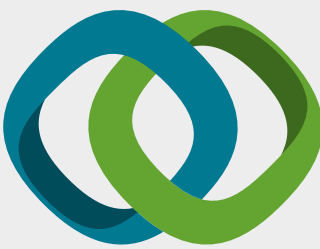

\section{Hindawi}

Submit your manuscripts at

www.hindawi.com
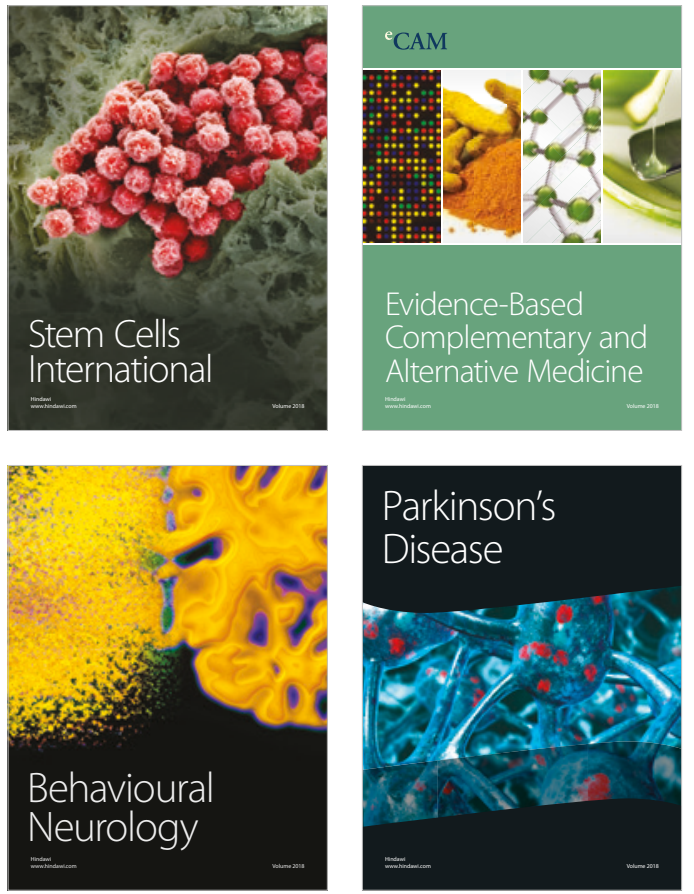

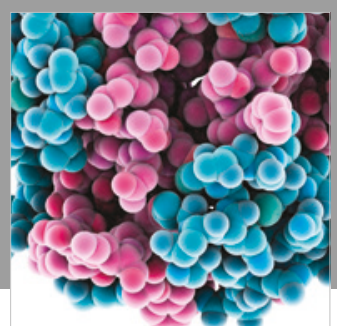

ournal of

Diabetes Research

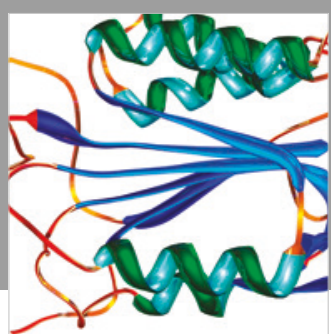

Disease Markers
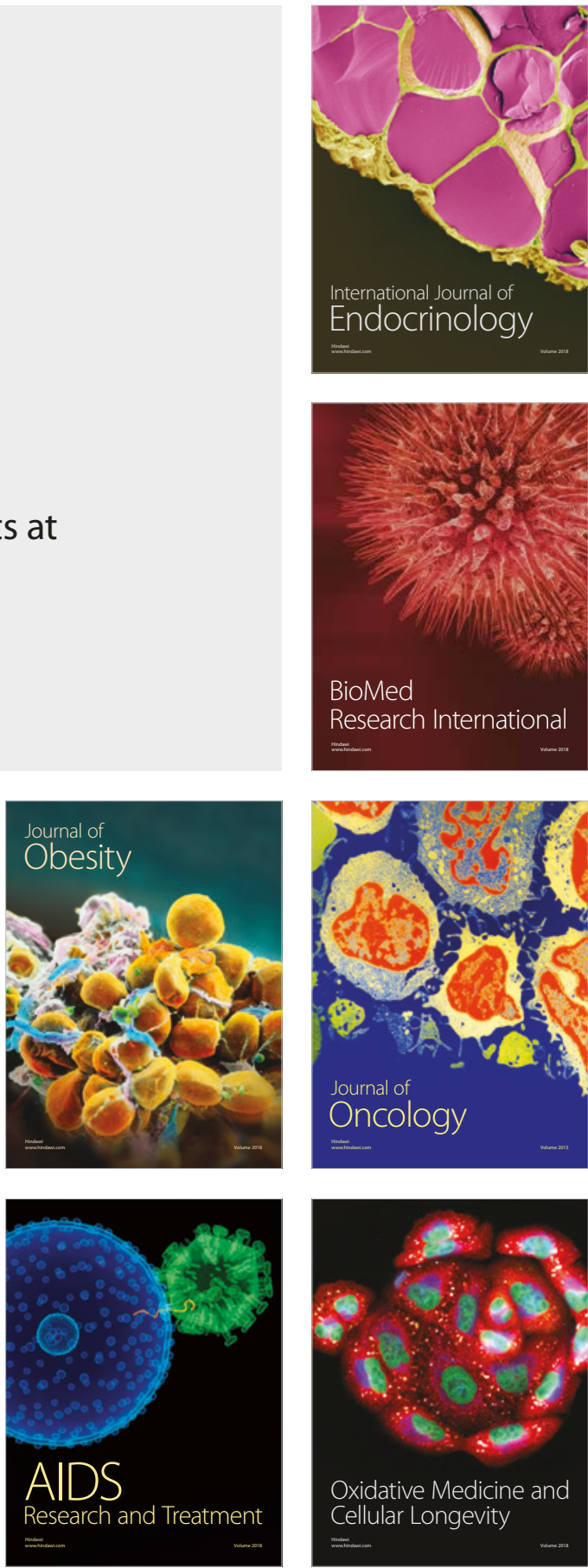